\title{
Role of diffusion-weighted MR imaging in diagnosis of acute pancreatitis
}

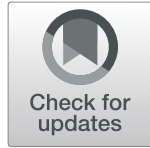

\author{
Hanan M. Abuzeid, Aya Yassin * (D), Omar F. Kamel and Kareem A. Sabry
}

\begin{abstract}
Background: The role of diffusion-weighted magnetic resonance imaging (DW-MRI) in the diagnosis of acute pancreatitis is assessed in this study by measuring the apparent diffusion coefficient (ADC) values in acute pancreatitis and comparing them with a control group. The aim of this study is to compare those two groups thus supporting the diagnosis of this disease.

Sixteen patients with acute pancreatitis and 16 control participants underwent diffusion-weighted imaging with $b$ values of 0,200 , and 800 . ADC maps were generated from the DW-MRI and ADC values, which were calculated for the pancreas, and the results of the two groups of patients were compared.

Results: The mean pancreatic ADC value in the acute pancreatitis group $\left(1.15 \times 10^{(-3)} \mathrm{mm}^{(2)} / \mathrm{s} \pm 0.28\right)$ was significantly lower than in the normal group $\left(1.6 \times 10^{-3} \mathrm{~mm}^{(2)} / \mathrm{s} \pm 0.2\right)$. A threshold ADC value of $1.38 \times 10^{-3}$ $\mathrm{mm}^{(2) / \mathrm{s}}$ yielded $81.25 \%$, specificity of $93.75 \%$, positive predictive value of $92.9 \%$, negative predictive value of $83.3 \%$, and accuracy of $91.8 \%$. Pancreatic ADC values were significantly lower in patients with acute pancreatitis than in the control group.

Conclusion: Diffusion-weighted magnetic resonance imaging could be an important supportive tool in the diagnosis of acute pancreatitis.
\end{abstract}

Keywords: Diffusion-weighted magnetic resonance imaging, DW-MRI, Apparent diffusion coefficient, ADC, Pancreas, Acute pancreatitis

\section{Background}

Acute pancreatitis is an acute non-infectious pancreatic inflammatory disorder. It occurs due to the activation of the pancreatic digestive enzymes and is normally a self-limited disease. About 20 to $30 \%$ of patients develop severe pancreatic necrosis, with morbidity and mortality rates of up to 20 to $45 \%$ [1]. Treatment of acute pancreatitis depends on the severity of the disease. In clinical practice, many laboratory findings and scoring systems have been used to predict the disease's course. Clinical signs, like epigastric pain, are non-specific and can be absent in up to $10 \%$ of patients. Serum lipase and amylase levels are also used to diagnose pancreatitis; however, they are within normal range in up to $20 \%$

* Correspondence: ayassin@hotmail.com

Faculty of Medicine, Ain Shams University, Cairo, Egypt of cases. It is therefore essential to grade acute pancreatitis radiologically [2].

Among the radiological studies, CT with contrast is the most prevalent modality used for disease staging and for determining severity. It is used in evaluation of the pancreatic parenchyma and detecting pancreatic and extra pancreatic inflammation, which is

Table 1 Demographic data of the control and patient group

\begin{tabular}{llll}
\hline & & $\begin{array}{l}\text { Control group } \\
\text { No. }=16\end{array}$ & $\begin{array}{l}\text { Patient group } \\
\text { No. }=16\end{array}$ \\
\hline Sex & Mean \pm SD & $38.56 \pm 16.22$ & $34.88 \pm 20.54$ \\
& Range & $18-66$ & $13-85$ \\
Age & Female & $12(75 \%)$ & $13(81.3 \%)$ \\
& Male & $4(25 \%)$ & $3(18.8 \%)$ \\
\hline
\end{tabular}


Table 2 Causes of pancreatitis in patient group by percentage

\begin{tabular}{lll}
\hline Cause & Idiopathic & $3(18.8 \%)$ \\
Gallstones & $11(68.8 \%)$ \\
Congenital (divisum) & $1(6.3 \%)$ \\
& latrogenic after ERCP & $1(6.3 \%)$ \\
\hline
\end{tabular}

closely linked to the severity of the course of the disease [3]. However, CT cannot be used in patients who have contraindications for iodinated contrast media, as it has been found to aggravate the disease and worsen the renal failure often caused by acute pancreatitis. MRI is also used, and it outweighs CT in diagnosis of the mild form of the disease, of peripancreatic fat infiltration, and of the pancreatic and biliary ducts' characterization. Using MRI is limited in emergency departments, however, because of its cost, limited availability, and long exam duration [4].

Diffusion-weighted magnetic resonance imaging (DW-MRI) is the best sequence that can be used during emergency settings because of its short acquisition time, the absence of radiation, and its high resolution $[5,6]$. The pancreas is both an exocrine and endocrine organ, and it therefore has a rich blood supply and is expected to have good diffusion characteristics. Acute pancreatitis can therefore be assessed by diffusion changes [7].

Studies reveal that DW-MRI restriction and low $\mathrm{ADC}$ values have the ability to assess pancreatic tissue inflammatory changes even in patients who do not have radiological findings using $\mathrm{CT}$ imaging. In DW-MRI, ADC values are used as a quantitative parameter, which reflects the microenvironment of diffusing water molecules [8].

In the current study, the main goal was to measure pancreatic ADC values in acute pancreatitis and to compare the results with a healthy control group.

Table 3 Relationship between mean ADC values of both groups

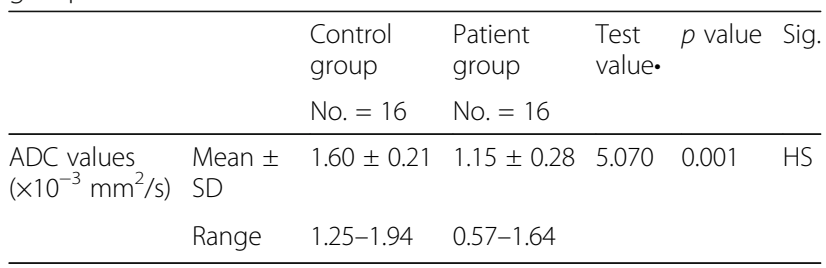

$p$ value $>0.05$ : non-significant (NS); $p$ value $<0.05$ : significant $(\mathrm{S}) ; p$ value $<$ 0.01: highly significant (HS)

- Independent $t$ test

\section{Methods}

This case-control study included 16 patients with acute pancreatitis referred from the emergency department of a university hospital, and 16 patients in a control group who were in the radiodiagnosis department for MRI examination. The cases were age- and sex-matched.

\section{Inclusion criteria}

- Patients with clinically and laboratory diagnosed acute pancreatitis.

- Both sexes included.

- No age predilection.

\section{Exclusion criteria}

- Patients known to have contraindications for MRI, e.g., an implanted magnetic device, pacemakers, or claustrophobia.

- Patients in a bad general condition needing life support.

\section{Procedure}

At the MRI unit, both groups were subject to: the following

- A full history-taking and clinical examination.

- An MRI examination, carried out at the MRI unit at the university hospital.

\section{Patient preparation}

- Prior to MRI examination, informed consent was obtained from the patient or the patient's parents or caregivers.

- No previous preparations were required.

- Detailed explanation of the imaging procedure was provided.

- The patient was positioned supine on the MRI table.

\section{Method of MRI examination}

The MRI study was performed using a $1.5 \mathrm{~T}$ machine (Achieva, Philips medical system, Eindhoven, Netherlands). A standard body coil was used for both conventional imaging and diffusion-weighted imaging.

\section{Examination protocol}

Morphological sequences were performed in multiple projections, including the following:

1. Axial, sagittal, and coronal T2-weighted turbo spin echo (T2 W TSE) images.

2. Axial and sagittal (STIR) images. 
Table 4 Relationship between sex/cause and mean ADC value in the patient group

\begin{tabular}{|c|c|c|c|c|c|c|}
\hline & & ADC values & & Test & & Sig. \\
\hline & & Mean \pm SD & Range & & & \\
\hline Sex & Female & $1.20 \pm 0.26$ & $0.57-1.64$ & $1.526 \bullet$ & 0.149 & NS \\
\hline & Male & $0.93 \pm 0.32$ & $0.57-1.16$ & & & \\
\hline Cause & Idiopathic & $1.36 \pm 0.18$ & $1.16-1.50$ & $8.438 \cdot$. & 0.003 & HS \\
\hline & Gallstones & $1.19 \pm 0.18$ & $0.97-1.64$ & & & \\
\hline & Congenital (divisum) & $0.57 \pm 0.00$ & $0.57-0.57$ & & & \\
\hline & latrogenic after ERCP & $0.57 \pm 0.00$ & $0.57-0.57$ & & & \\
\hline
\end{tabular}

$p$ value $>0.05$ : non-significant (NS); $p$ value $<0.05$ : significant (S); $p$ value $<0.01$ : highly significant (HS)

Independent $t$ test

..One-way ANOVA test

3. Axial T1-weighted turbo spin echo (T1W TSE) images.

4. Axial DWI using a multi-slice single-shot echoplanar imaging (EPI) sequence with different $b$ values.

5. ADC maps in grayscale were automatically produced, with the applied mono-exponential decay model including all $b$ values $(0,200$, and 800$)$.

\section{Duration of the examination}

The MRI took about 15-20 min.

\section{Image analysis}

In the control group, mean $\mathrm{ADC}$ values from each sector (head, body, and tail) with a minimum area of $80 \mathrm{~mm}^{2}$ were calculated for each patient directly from the ADC map data on the workstation. In the acute pancreatitis group, the ADC from a single (region of interest) ROI with the highest signal

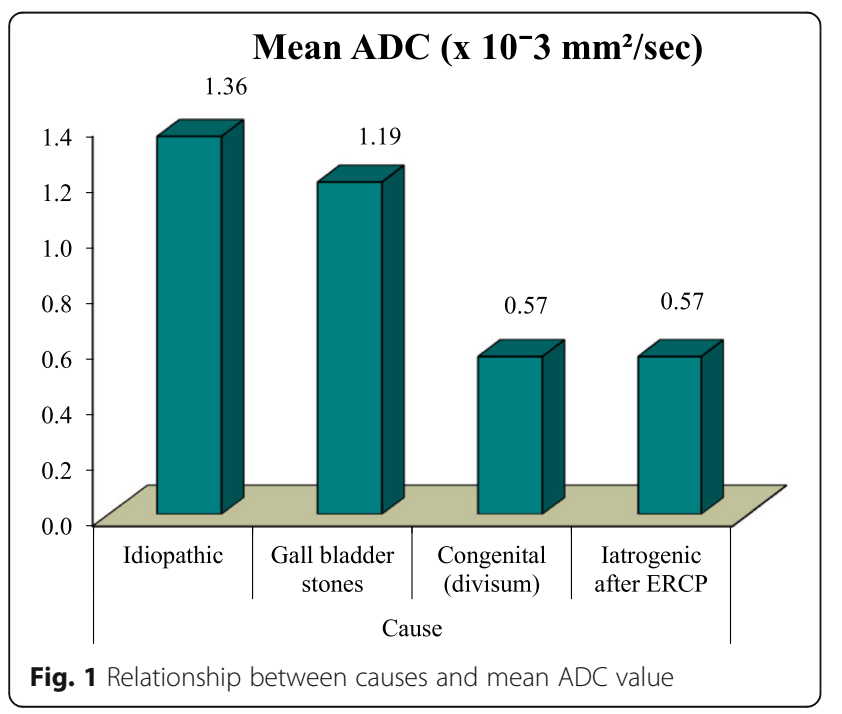

intensity in the head, body, or tail with a minimum area of $90 \mathrm{~mm}^{2}$ was measured. Areas with pancreatic fluid, pancreatic duct, pseudocysts, cystic lesions, and artifacts were excluded from the region of interest.

The images were analyzed by a senior radiologist who has 4 years of body MRI experience and who was not blinded to clinical data.

The diagnosis was confirmed by the clinical picture and the laboratory tests.

\section{Statistical analysis}

Data were coded using IBM SPSS (version 23). The quantitative data were expressed as mean, SD, and ranges when their distribution found parametric. The qualitative data were expressed as numbers and percentages.

The comparison between the quantitative data and parametric distribution of the two groups was done using the independent $t$ test; the comparison between groups regarding qualitative data was done by using the chi-square test; and the comparison between more than two groups was done using one-way ANOVA.

Spearman's rank correlation coefficient was used to assess the correlation between two quantitative parameters in the same group.

A receiver operating characteristic (ROC) curve was used in the quantitative form to determine sensitivity,

Table 5 Diagnostic performance of ADC value $\times 10-3$ in discrimination of acute pancreatitis and normal

\begin{tabular}{|c|c|c|c|c|c|c|}
\hline Parameter & AUC & $\begin{array}{l}\text { Cut-off } \\
\text { Point }\end{array}$ & Sensitivity & Specificity & PPV & NPV \\
\hline $\begin{array}{l}\text { Mean ADC }\left(\times 10^{-3}\right. \\
\left.\mathrm{mm}^{2} / \mathrm{s}\right)\end{array}$ & 0.918 & $\leq 1.32$ & 81.25 & 93.75 & 92.9 & 83.3 \\
\hline
\end{tabular}




\begin{tabular}{|c|c|c|c|c|c|c|}
\hline Parameter & AUC & Cut-off Point & Sensitivity & Specificity & PPV & NPV \\
\hline & & & & & & \\
\hline $\begin{array}{c}\text { Mean ADC } \\
\left(x 10^{-3} \mathrm{~mm}^{2} / \mathrm{sec}\right)\end{array}$ & 0.918 & $\leq 1.32$ & 81.25 & 93.75 & 92.9 & 83.3 \\
\hline
\end{tabular}

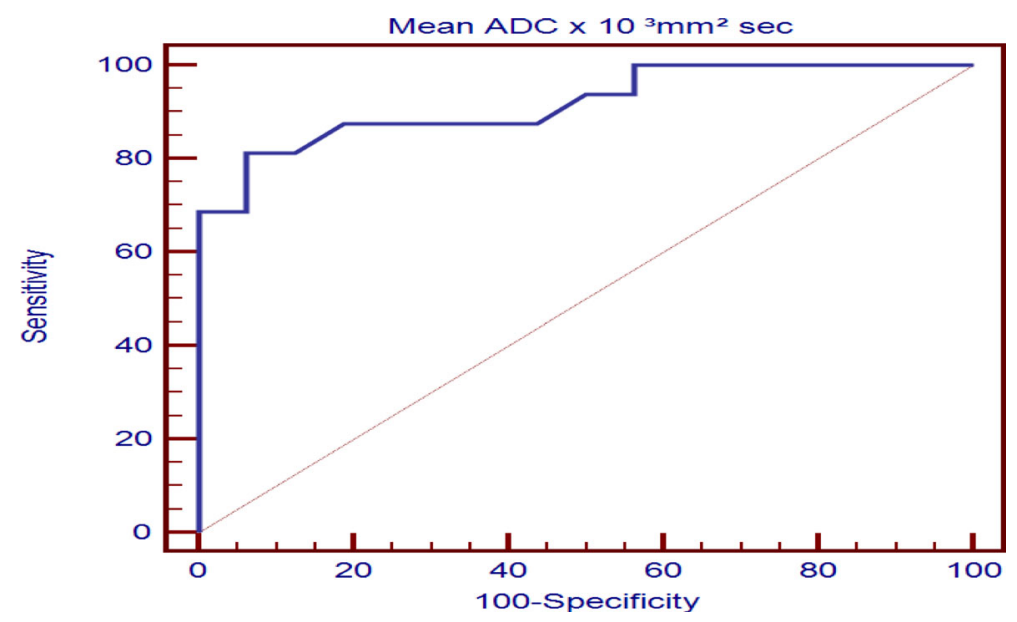

Fig. 2 ROC curve between control and patient group regarding mean ADC value

specificity, positive predictive value (PPV), negative predictive value (NPV), and the cut-off point of mean ADC. The confidence interval was set to $95 \%$ and the margin of error accepted was set to $5 \%$. The significance of $p$ values was established as follows:

$p$ value $>0.05$ : non-significant $(\mathrm{NS})$

$p$ value $<0.05$ : significant $(\mathrm{S})$

$p$ value $<0.01$ : highly significant $(\mathrm{HS})$

\section{Results}

This case-control study was conducted on 16 patients who had presented with acute pancreatitis and had been referred to an MRI unit at a university hospital. The most restricted pancreatic area was assessed in each patient. We included a total of 16 patients and compared them to 16 control cases who had an abdominal pathology that was not pancreatic.

The data was collected, analyzed, and tabulated as follows:

\section{Demographic distribution of study population}

The control group ranged in age from 18 to 66 years old with a mean of $38.56 \pm 16.2$ years. Men made up $25 \%$ of the control group and women $75 \%$. Patients were aged between 13 and 85 years old with a mean of $34.88 \pm 20.54$ years; men made up $19 \%$ of the patient group and women $81 \%$ (Table 1).

\section{Distribution of study population according to causes}

In the 16 patients with acute pancreatitis, the etiology was biliary in $68.8 \%(11 / 16)$, congenital in $6.3 \%(1 /$ $16)$, and iatrogenic in $6.3 \%(1 / 16)$, while $18.8 \%(3 / 16)$ of the patients did not have a specified etiology (Table 2).

Distribution of study population according to ADC results The mean pancreatic ADC value in the acute pancreatitis group $\left(1.15 \times 10^{(-3)} \mathrm{mm}^{(2)} / \mathrm{s} \pm 0.28\right)$ was significantly lower than in the normal group $(1.6 \times$ $10^{(-3)} \mathrm{mm}^{(2)} / \mathrm{s} \pm 0.2$ ) (Table 3). There was no significant difference between sex and mean ADC value of pancreatitis in the patient group (Table 4, Fig. 1).

The receiver operating characteristics (ROC) curve was used to define the best cut-off ADC value $\times 10^{-3}$ for differentiation of acute pancreatitis and normal pancreas, which was $\leq 1.32$, with sensitivity of $81.25 \%$, specificity of $93.75 \%$, positive predictive value 

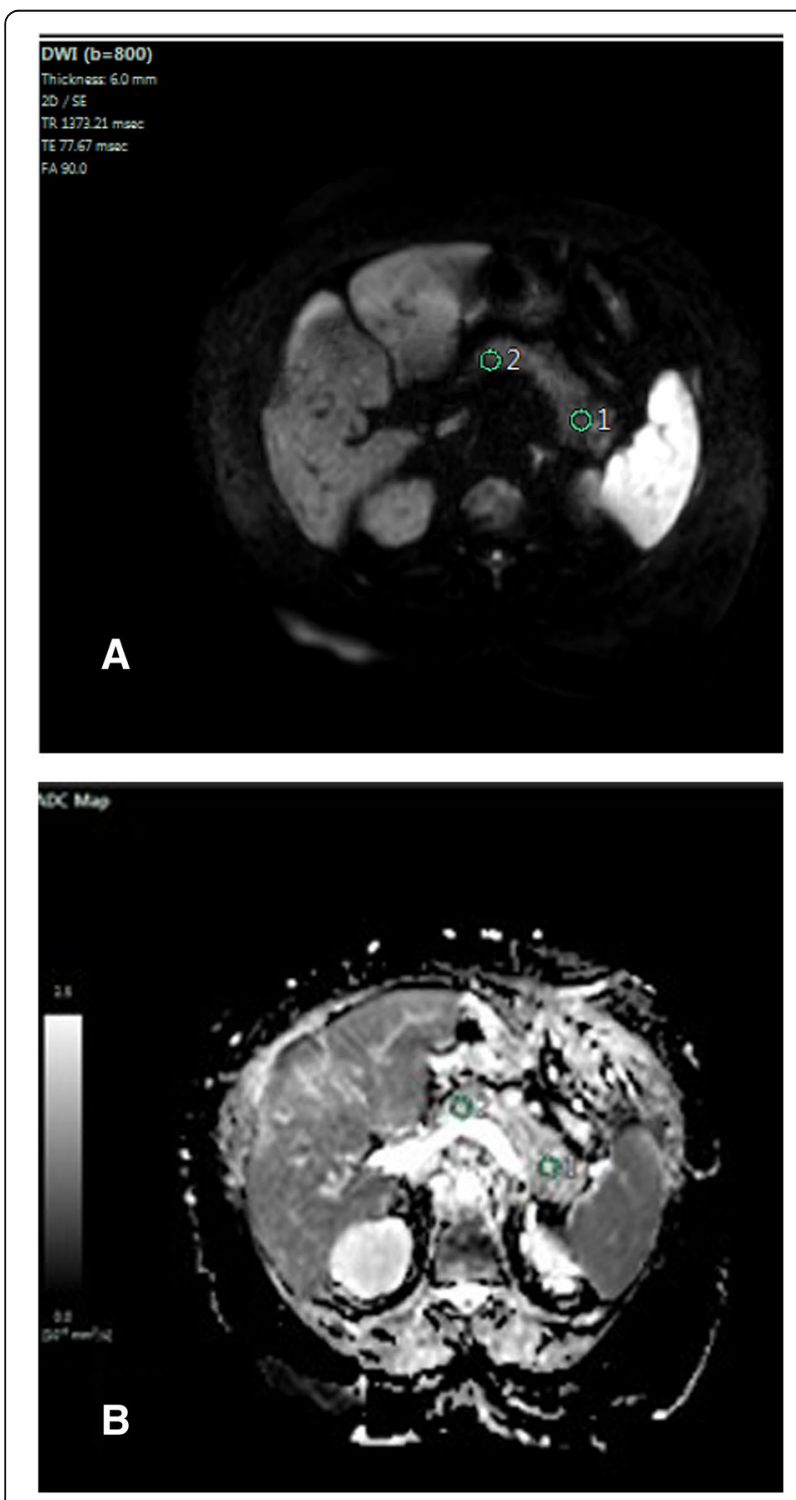

Fig. 3 DWI showing no restriction of pancreatic parenchyma and high $A D C$ value of pancreas on $A D C$ map of $92.9 \%$, negative predictive value of $83.3 \%$, and accuracy of $91.8 \%$ (Table 5, Fig. 2).

These are all well-demonstrated in Figs. 3, 4, 5, 6, and 7.

Table 1 shows the demographic data of both control and patient groups.

Table 2 shows the causes of pancreatitis in the patient group. Gallstones were the most common cause in our patient group.

Table 3 shows the relationship between mean ADC values between both groups, showing significant difference between both groups being much lower in acute pancreatitis than the control group.

Table 4 shows that there is no significant difference between sex and mean ADC value of pancreatitis in the patient group. It also shows a strong relationship between the cause of pancreatitis and the mean $\mathrm{ADC}$ value; mean $\mathrm{ADC}$ values are significantly lower in cases with congenital and iatrogenic causes, but not in the case of gallstones, as illustrated in Fig. 1.

Table 5 and Fig. 2 show a receiver operating characteristics (ROC) curve that was used to define the best cutoff $\mathrm{ADC}$ value $\times 10-3$ for differentiation of acute pancreatitis and normal pancreas, which was $\leq 1.32$, with sensitivity of $81.25 \%$, specificity of $93.75 \%$, positive predictive value of $92.9 \%$, negative predictive value of $83.3 \%$, and accuracy of $91.8 \%$

\section{Discussion}

As discussed above, MRI is considered to be the modality of choice to assess pancreatic necrosis and severity as a result of severe pancreatitis, which has a high mortality rate [9]. The pathogenesis of acute pancreatitis is likely due to acinar cell death, infiltration of acinar spaces by polymorphonuclear leucocytes, deposition of fibrin in intercellular spaces, and microthrombi in blood vessels. The areas of restricted diffusion show higher signal intensity on DW-MRI with low ADC values [10]. DW-MRI has been suggested as a helpful tool for detecting acute pancreatitis since inflammatory processes are related to restricted water diffusion [11]. CT imaging with IV contrast medium in severe acute pancreatitis patients who have renal failure may increase the severity of the disease [12]. In addition, CT is usually delayed until the third and the fourth day after the onset of the disease, while DW-MRI can be used in the first 3 days to detect early changes in the disease $[13,14]$. DW-MRI is also an outstanding imaging tool for patients with a history of allergy to IV contrast medium $[15,16]$.

This study included 16 patients with acute pancreatitis and 16 healthy controls. The mean pancreatic ADC in 


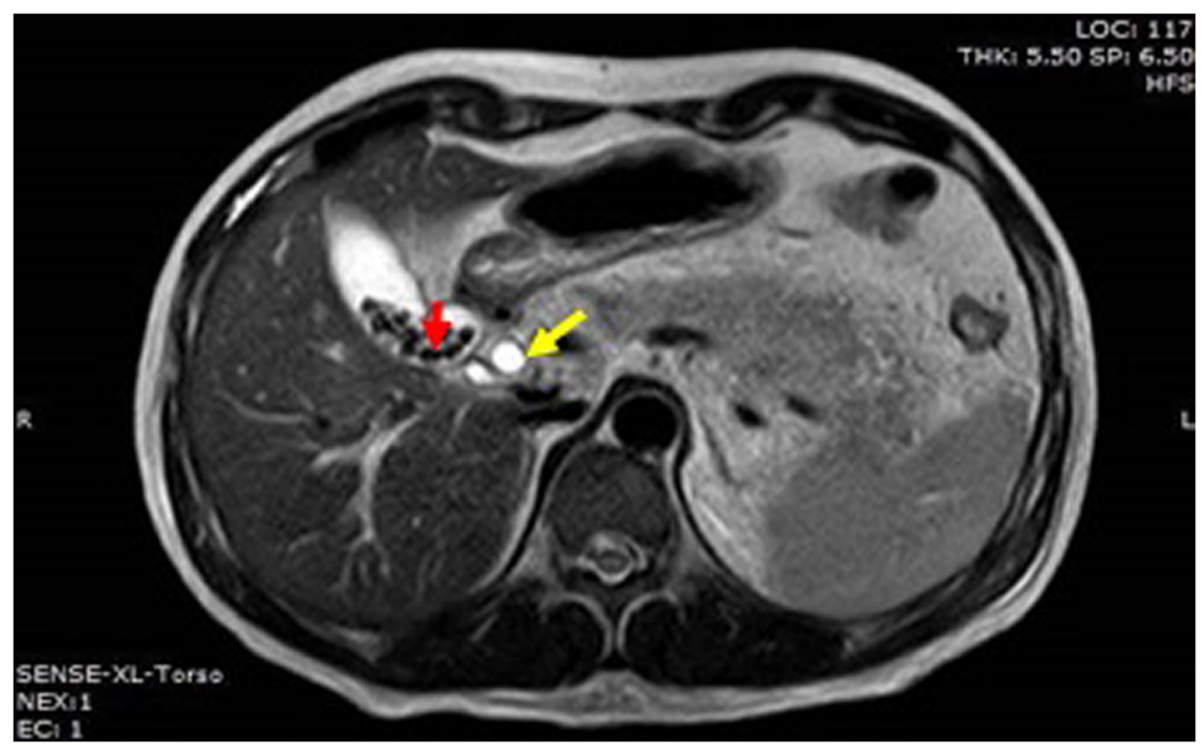

Fig. 4 Axial T2WI shows calcular gallbladder (red arrow) with dilated CBD (yellow arrow)

the pancreatitis group $\left(1.15 \times 10^{(-3)} \mathrm{mm}^{(2)} / \mathrm{s} \pm 0.28\right)$ was significantly lower than in the control group $(1.6 \times$ $\left.10^{(-3)} \mathrm{mm}^{(2)} / \mathrm{s} \pm 0.21\right)$. In the control group, the average $\mathrm{ADC}$ values in the tail were lower than in the head and body.

The study showed the cut-off value to be $1.32 \times$ $10^{(-3)} \mathrm{mm}^{2} / \mathrm{s}$, quite similar to those in the study done by Salik et al. [17], who showed the threshold total ADC value that best separated the acute pancreatitis and control groups was $1.60 \times 10^{-3}$ $\mathrm{mm}^{2} / \mathrm{s}$.

The cases studied showed that MRI is also beneficial in evaluating numerous findings such as gallstones, peripancreatic fluid, necrosis, and lymphadenopathy. Diffusion also differentiates between normal and abnormal pancreatic tissue and thus is well-correlated to clinical and laboratory findings. This was also proved in the research by Wiggermann et al. [18].

The pancreatic mean ADC value in the acute pancreatitis group was significantly lower than in the control group, at $1.15 \times 10^{(-3)} \mathrm{mm}^{(2)} / \mathrm{s}$ in the former compared to $1.6 \times 10^{(-3)} \mathrm{mm}^{(2)} / \mathrm{s}$ in the latter. This is also similar to the results of a study done by Xinghui et al. [19].

The best ADC threshold value for distinguishing acute pancreatitis from a healthy pancreas was 1.32 $\times 10^{-3} \mathrm{~mm}^{2} / \mathrm{s}$, with a sensitivity of $81.25 \%$, specificity of $93.75 \%$, positive predictive value of $92.9 \%$, negative predictive value of $83.3 \%$, and accuracy of $91.8 \%$.

This study has some limitations, including the fact that it is a small case-control study. Despite care being taken to avoid artifacts and optimizing the protocol to reduce imaging time and motion, MR artifacts introduce unavoidable ADC measurement errors, especially in body imaging applications. Sampling errors were reduced by increasing the area of the ROI in the pancreatitis group. A second limitation was increasing the $b$ value in order to improve the sensitivity of diffusion, leading to the reduction of the signal to noise ratio (SNR), which affects ADC measurement. In the current study, a maximum $b$ value of $800 \mathrm{~mm}^{2} / \mathrm{s}$ was used. We also had a limitation of having just one radiologist reviewing the images. Two readers with analysis of agreement and discrepancy will be used in future studies with greater numbers of patients.

\section{Conclusion}

An acutely inflamed pancreas demonstrates restricted diffusion and can be differentiated from a normal pancreas based on DW-MRI findings through increased signal in DW-MRI and decreased ADC values. Future studies for evaluating the diagnostic accuracy of DW-MRI and investigating the role of ADCs as a prognostic indicator in acute pancreatitis may be helpful. 


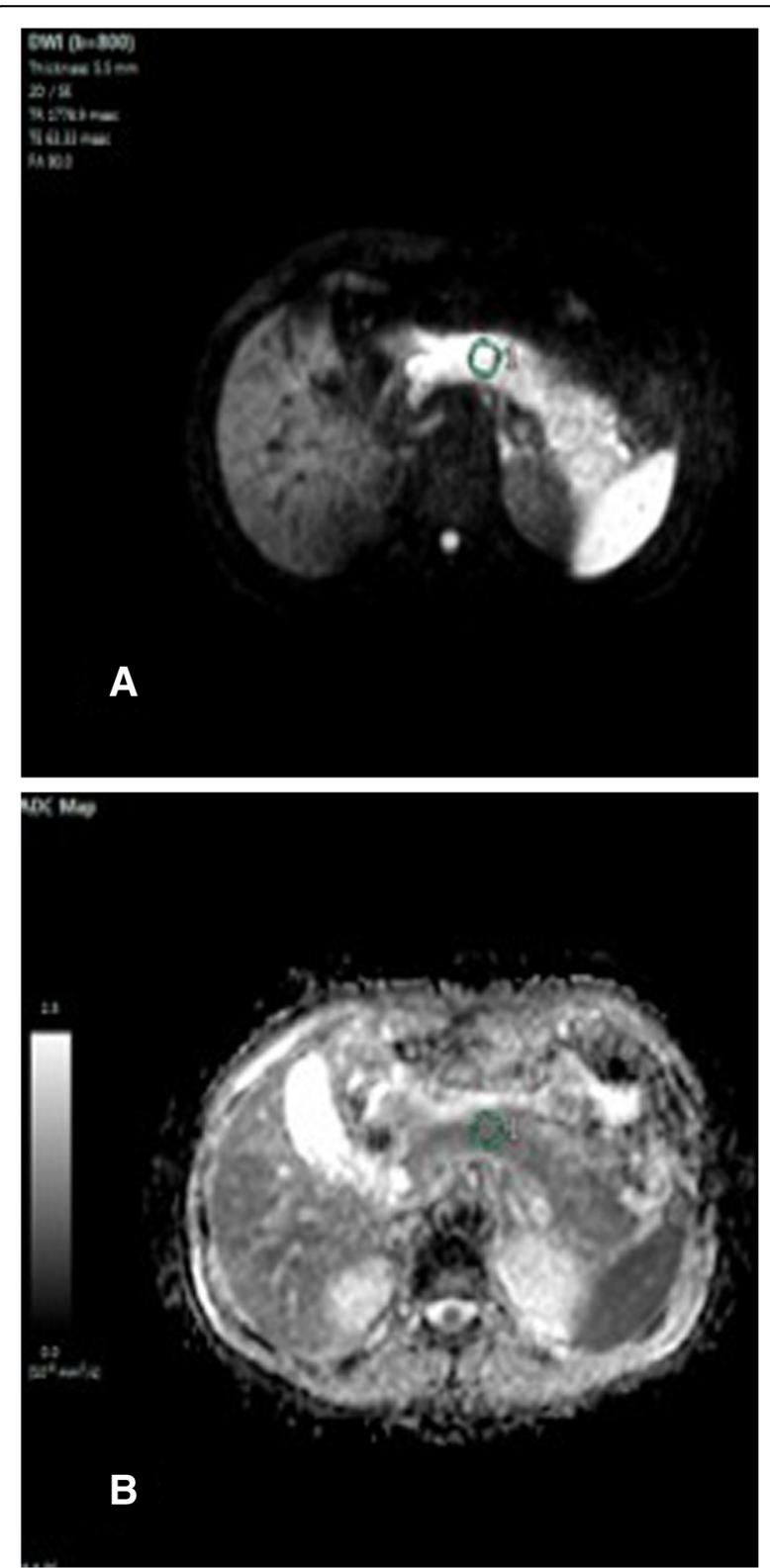

Fig. 5 DWI showing restricted pancreas and low ADC value $1.01 \times$ $10^{-3} \mathrm{~mm}^{2} / \mathrm{s}$ of pancreatic body on ADC map 


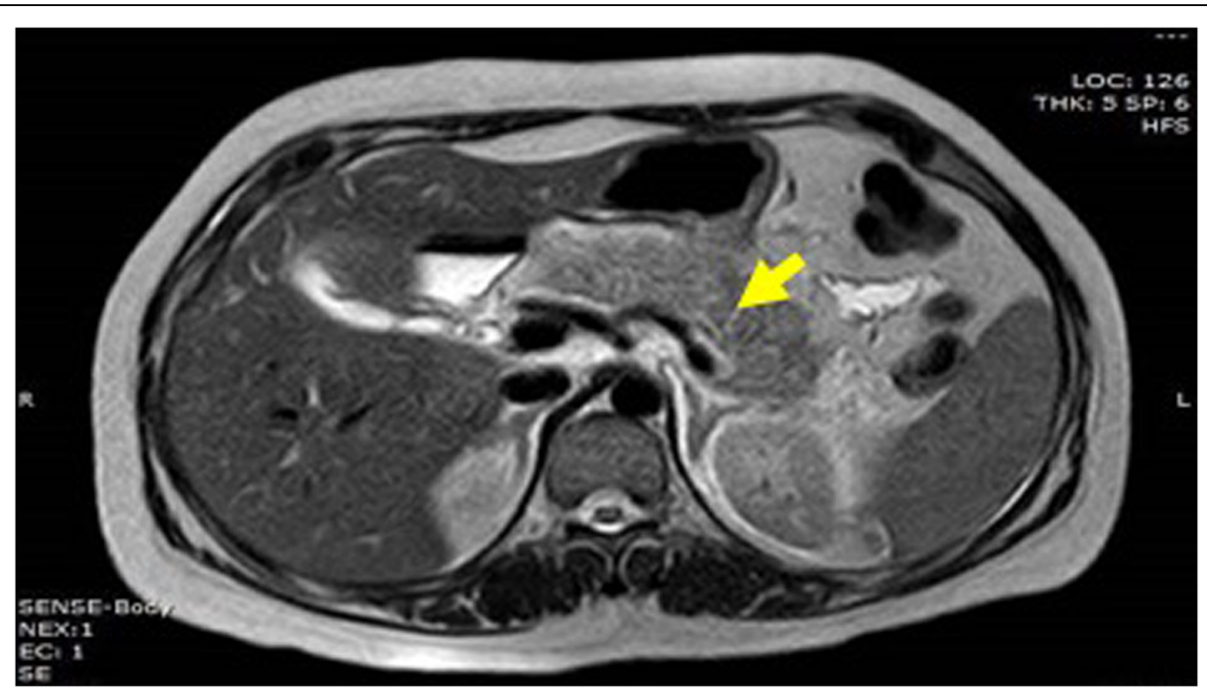

Fig. 6 Axial T2WI shows edematous pancreas (yellow arrow) with minimal peripancreatic fluid
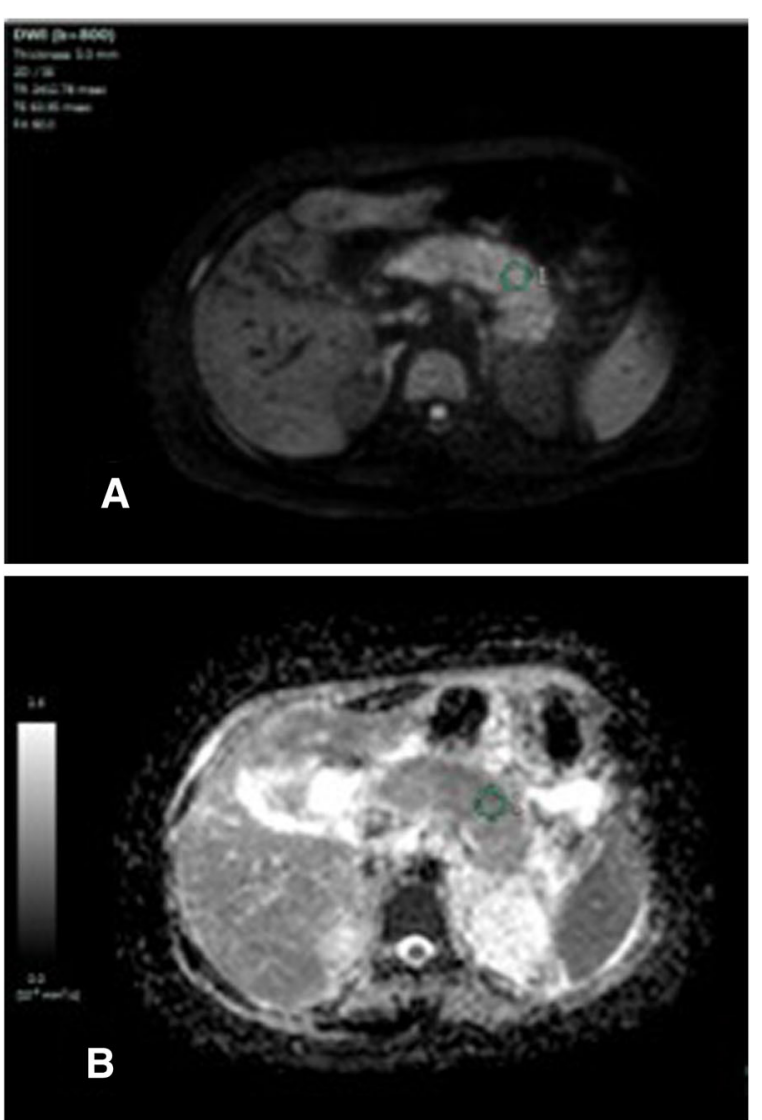

Fig. $7 \mathrm{DW}$ showing restricted pancreas and low ADC value of pancreatic body on ADC map $0.87 \times 10^{-3} \mathrm{~mm}^{2} / \mathrm{s}$ 


\section{Abbreviations}

ADC: Apparent diffusion coefficient; AP: Acute pancreatitis; AUC: Area under the curve; CT: Computed tomography; DWI: Diffusion-weighted imaging; DW-MRI: Diffusion-weighted magnetic resonance imaging; FSE: Fast spin echo; HS: Highly significant; ROI: Region of interest; MRI: Magnetic resonance imaging; NPV: Negative predictive value; NS: Non-significant; PPV: Positive predictive value; ROC: Receiver operating characteristic; S: Significant; SD: Standard deviation; SNR: Signal to noise ratio; SPSS: Statistical Package for Social Science; TSE: Turbo spin echo

\section{Acknowledgements}

Our sincere thanks to all our professors and colleagues in the Radiology Department, Faculty of Medicine, Ain Shams University.

\section{Authors' contributions}

H.M.A participated in the design of the study, image interpretation, and data collection. A. Y. put the idea of research and shared in the design of the study, image interpretation, and data collection as well as the submission and follow-up. O.F.K participated in the design of the study, image interpretation, and data collection. K.A.S put the idea of the study, editor of the manuscript, image interpretation, and performed the statistical analysis. All authors read and approved the final manuscript

\section{Funding}

Not applicable (no funding received for this study).

\section{Availability of data and materials}

All the datasets used and analyzed in this study are available with the corresponding author on reasonable request.

\section{Ethics approval and consent to participate}

Written informed consent was signed by all patients before the examination. The study was approved by the Research Ethics Committee (REC) of the Faculty of Medicine, Ain Shams University, FWA 00017585 (approval number 234/2017) and experiments were conducted in accordance with the ethical guidelines

\section{Consent for publication}

All patients included in this research were fully conscious and older than 16 year old and gave written informed consent to publish the data contained within this study.

\section{Competing interests}

The authors declare that they have no competing interests.

Received: 5 October 2019 Accepted: 26 June 2020

Published online: 06 October 2020

\section{References}

1. Busireddy KK, Al Obaidy M, Ramalho M et al (2014) Pancreatitis imaging approach. World jo, of gastrointestinal pathophysiology. 5(3):252-270

2. Yencilek $\mathrm{E}$, Telli S, Tekesin $\mathrm{K}$ et al (2014) The efficacy of diffusion weighted imaging for detection of acute pancreatitis and comparison of subgroups according to Balthazar classification. Turk J Gastroenterol 25:553-557

3. Pongprasobchai S, Vibhatavata P, and Apisarnthanarak P (2017): Severity, Treatment, and Outcome of Acute Pancreatitis in Thailand: The First Comprehensive Review Using Revised Atlanta Classification, . 2450-0455.

4. Bollen L.T (2016).: Imaging Assessment of Etiology and Severity of Acute Pancreatitis American pancreatic association Version 1.0, November 1.

5. Aydin H, Tatar G.l., Hekimoglu B (2014).: The Role of Diffusion Weighted MR Imaging in the Diagnosis of Acute Pancreatitis. International Journal of Emergency Mental Health and Human Resilience, Vol. 16, No.93-98,15224821.

6. Rege NR, Sharma P, Naikwadi A et al (2017) The Potential Role of Apparent Diffusion Coefficient Values in the Differentiation of Tuberculous and Malignant Lymph Nodes of Neck - In Correlation with Histopathology. JMSCR, Vol 5(May):324-337

7. Nissan N, Golan T, Furman-Haran E et al. (2014): Diffusion tensor magnetic resonance imaging of the pancreas. PloS one:;9(12).

8. Balthazar EJ, Robinson DL, Megibow AJ et al (1990) Acute pancreatitis: Value of CT in establishing prognosis. Radiology 174:331-336
9. Thomas S, Kayhan A, Lakadamyali $\mathrm{H}$ et al (2012) Diffusion MRI of acute pancreatitis and comparison with normal individuals using ADC values, Emerg Radiol. Jan 19(1):5-9

10. Sugimoto $\mathrm{M}$, Takada T, Yasuda $\mathrm{H}$ et al. (2006) The lethal toxicity of pancreatic ascites fluid in severe acute necrotizing pancreatitis. Hepatogastroenterology.53:442-446

11. Guo Y, Cai YQ, Cai ZL et al (2002) Differentiation of clinically benign and malignant breast lesions using diffusion-weighted imaging. J Magn Reson Imaging. 16:172-178

12. Hocaoglu E, Aksoy S, Akarsu C et al. (2015) Evaluation of diffusion-weighted MR imaging in the diagnosis of mild acute pancreatitis. Clin Imaging:463467

13. In Chul Nam, Seung Ho Kim, Seon-Jeong Kim (2016) "Added Value of Diffusion Weighted Imaging for Detecting Pancreatic Abnormality in Patients with Clinically Suspected Acute Pancreatitis", iMRI:241-249

14. Lee NK, Kim S, Kim GH et al. (2012). Diffusion-weighted imaging of biliopancreatic disorders: correlation with conventional magnetic resonance imaging. World J Gastroenterol:4102-4117

15. Shinya S, Sasaki T, Nakagawa Y et al. (2009) The efficacy of diffusionweighted imaging for the detection and evaluation of acute pancreatitis. Hepatogastroenterology:1407-1410.

16. Carmona-Sanchez R, Uscanga L, Bezaury et al. (2000) Potential harmful effect of iodinated intravenous contrast medium on the clinical course of mild acute pancreatitis. Arch Surg.; 135:1280-1284.

17. Salik AE, Islim F, Tekdos $Y$ et al (2013) The diagnostic role of DW-MRI in acute pancreatitis. Assessment of the clinical outcome, Congress: ECR 2013 6(5):164-173

18. Wiggermann P, Grutzmann R, Weissenböck A et al (2012) Apparent diffusion coefficient measurements of the pancreas, pancreas carcinoma, and mass-forming focal pancreatitis. Acta Radiologica 53:135-139

19. Xinghui Li, Ling Zhuang, Xiaoming Zhang et al. (2016): Preliminary Study of MR Diffusion Tensor Imaging of Pancreas for the Diagnosis of Acute Pancreatitis. September 1 Sep 1:11(9):e0160115

\section{Publisher's Note}

Springer Nature remains neutral with regard to jurisdictional claims in published maps and institutional affiliations.

\section{Submit your manuscript to a SpringerOpen ${ }^{\circ}$ journal and benefit from:}

- Convenient online submission

- Rigorous peer review

- Open access: articles freely available online

High visibility within the field

- Retaining the copyright to your article

Submit your next manuscript at $\boldsymbol{\nabla}$ springeropen.com 